\title{
RECEITAS DE PATROCÍNIO E PUBLICIDADE NOS CLUBES DE FUTEBOL: UMA ANÁLISE SOB A ÓTICA DAS REDES SOCIAIS
}

\section{SPONSORSHIP AND ADVERTISING REVENUE IN FOOTBALL CLUBS: AN ANALYSIS FROM THE PERSPECTIVE OF SOCIAL NETWORKS}

\author{
Sponsorship and Advertising Revenue in Football Clubs: an Analysis From the Perspective of Social Networks
}

\section{RESUMO}

Este estudo objetiva verificar a relação entre a quantidade de seguidores nas redes sociais (Facebook, Instagram, Twitter e Youtube) dos clubes e a captação de recursos de patrocínio e publicidade, no pressuposto de que a presença dos torcedores nas redes sociais seria indicativo de um público engajado com o clube e interessado nas suas atividades e, possivelmente, propenso a consumir produtos ou adquirir marcas dos patrocinadores parceiros. Foram analisados 32 clubes entre 2016 e 2017 que disponibilizaram suas demonstrações contábeis e constavam na análise de rankings digitais elaborada pelo Ibope Repucom. Utilizou-se como modelo econométrico a regressão de dados em painel com abordagem efeitos aleatórios. Quanto aos resultados, as variáveis rede social e tamanho mostraram-se positivamente significativas, confirmando a hipótese de relação positiva entre a receita de patrocínio e publicidade e o engajamento dos clubes nas redes sociais, demonstrando que os clubes devem aprimorar-se em formas de atrair visibilidade para seus perfis nas redes, permitindo que seus patrocinadores fiquem mais interessados, de modo que essa fonte de receita possa elevar-se frente a nova realidade de exposição de imagem dos clubes. Adicionalmente, avaliou-se se uma rede social possuía maior influência na captação de recursos de patrocínio e publicidade comparadas a outras e verificou-se que com exceção do Youtube, as demais rede sociais analisadas individualmente foram positivamente significativas e com coeficientes semelhantes entre si, ressaltando a importância dos clubes estarem presentes em todas as redes sociais, pois de forma conjunta há maximização de visibilidade, tornando-se mais atrativa para angariar montantes maiores de receita de patrocínio e publicidade.

Palavras chave: Clubes de futebol, Receitas de Patrocínio e Publicidade, Rede Social.

\begin{abstract}
This study aims to verify the relationship between the presence of fans on social networks (Facebook, Instagram, Twitter and Youtube) and fundraising sponsorship and advertising, on the assumption that the presence of fans on social networks would be indicative of an engaged audience with the club and interested in its activities and possibly prone to consume products or acquire brands from the sponsoring partners. We analyzed 32 clubs between 2016 and 2017 that provided financial statements and were included in the digital rankings analysis prepared by Ibope Repucom. The econometric model used was panel data regression with random effects approach. Regarding the results, the variables social network and size were positively significant, confirming the hypothesis of a positive relationship between sponsorship and advertising revenue and the engagement of clubs in social networks, showing that clubs should improve in ways to attract fans to their profiles on the net, allowing their sponsors to become more visibility, so that this source of revenue can increase as a result the new reality of club image exposure. Additionally, it was evaluated if a social network had greater influence on sponsorship and advertising fundraising than others and it was found that all social network variables analyzed individually were positively significant and with similar coefficients, highlighting the importance of clubs to be present in all social networks, because together there is maximization of visibility, making it more attractive to raise larger amounts of sponsorship and advertising revenue. Keywords: Football Clubs, Sponsorship and Advertising Revenue, Social Network.
\end{abstract}

\author{
Thais Azzolini Piva \\ Doutoranda e Mestre em Ciências \\ Contábeis pela Universidade Federal do Rio \\ de Janeiro (UFRJ). Graduada em Ciências \\ Contábeis pelo Instituto Brasileiro de \\ Mercado de Capitais (IBMEC). Graduada \\ em Administração pela Universidade \\ Federal Rural do Rio de Janeiro (UFRRJ). \\ Contato: Av. Pasteur, 250, Sala 250, \\ Campus Praia Vermelha, Urca, Rio de \\ Janeiro, RJ, CEP: 22.290-240. E-mail: \\ thaispiva03@hotmail.com
}

\section{Odilanei Morais dos Santos Doutor em Contabilidade pela Universidade de São Paulo (USP). Mestre em Ciências Contábeis pela Universidade Federal do Rio de Janeiro (UFRJ). Graduado em Ciências Contábeis pela Universidade Federal do Pará (UFPA). Docente do curso de Ciências Contábeis na Universidade Federal do Rio de Janeiro (UFRJ). Contato: Av. Pasteur, 250, Sala 250, Campus Praia Vermelha, Urca, Rio de Janeiro, RJ, CEP: 22.290-240. E-mail: profodilanei@gmail.com}

crofodianei@gmailcom 


\section{INTRODUÇÃO}

O futebol brasileiro movimenta montantes significativos de receitas todos os anos. Somente em 2017, por exemplo, foram arrecadados R \$ 6,25 bilhões, considerando os clubes, as 27 federações estaduais e a CBF, sendo $81 \%$ referentes aos vinte maiores clubes (Sports Value, 2018). Um aspecto crucial para o sucesso dos clubes consiste na busca por formas de diversificação de suas fontes de receitas (Miragaia, Ferreira \& Ratten, 2017).

Segundo Silva e Campos Filho (2006), foi a partir da Lei Pelé, em 1998, que houve a necessidade de fontes alternativas de receita, pois o passe dos jogadores foi extinto e esta era a principal fonte de receita dos clubes até então. Nesse sentido, cabe ressaltar que umas das principais fontes alternativas é a arrecadação de receitas de patrocínio e publicidade, que tem se destacado a cada ano e são definidas como o investimento feito por uma empresa em uma entidade desportiva a fim de atrair maior atenção para sua marca ao se vincular a um clube (Morgan \& Summers, 2008).

Esse tipo de receita vem ganhando destaque a cada ano, já que, conforme a análise feita pelo Itaú BBA (2018), a receita de patrocínio dos clubes analisados aumentou $27 \%$ em 2017, chegando à marca de $\mathrm{R} \$ 734$ milhões, o que confirma o crescente interesse das organizações nesse tipo de investimento. Isso decorre do fato de que as organizações possuem benefícios ao investirem no patrocínio das entidades desportivas, tais como: sua imagem passa a se destacar frente a outras empresas, seus produtos passam a ser reconhecidos, há incremento de vendas tanto no curto como no longo prazo (Cardia, 2004).

Para auxiliar nesse processo, atualmente existem as redes sociais que promovem uma nova forma de comunicação e interação entre os indivíduos (Brogan, 2012). Nesse caso, elas possibilitam uma nova abordagem de exposição de marcas patrocinadoras.

Isso ocorre, pois toda e qualquer informação compartilhada pelos clubes por meio destas ferramentas acaba sendo visualizada por milhões de seguidores, que são um dos stakeholders chave desse setor. Nesse contexto, Mangold e Faulds (2009) afirmam que a partir das redes sociais consumidores em potencial passam a tomar conhecimento sobre determinada marca e a vinculação desta com algo que os agrade influencia na sua decisão de compra.

Corroborando com essa visão, Salles e Castro Junior (2015) destacam que as redes sociais possuem caráter mercadológico, exigindo que os clubes se aprofundem no que diz respeito a utilização destas, buscando estratégias como forma de atrair patrocinadores a partir da visibilidade que possuem.

Para demonstrar o grande alcance das redes sociais, evidencia-se, em caráter ilustrativo, que somente no Facebook, em 2017, os quarenta clubes com maior número de seguidores totalizavam 68.372.766 torcedores-seguidores (Ibope Repucom, 2017). Ou seja, as redes sociais podem atuar como grandes aliadas, tanto na captação de recursos quanto na comunicação com os torcedores.

Diante disso, busca-se tratar do seguinte problema: Qual a relação entre a quantidade de seguidores nas redes sociais e a captação de recursos de patrocínio e publicidade?

O objetivo é verificar a relação entre a quantidade de seguidores nas redes sociais e a captação de recursos de patrocínio e publicidade, pois os clubes competem entre si por empresas interessadas em investir no futebol, sendo fundamental a busca pela identificação da motivação para esse investimento. Lobato, Dias e Teixeira (2012) destacam que os clubes têm adotado novas estratégias para compor suas receitas e melhorar sua gestão financeira, promovendo maior conscientização sobre a figura do torcedor e o considerando sua razão de existir.

Sendo assim, a presença de um maior número de seguidores nas redes sociais pode identificar um público engajado com o clube e interessado nas suas atividades e, possivelmente, propenso a consumir produtos ou adquirir marcas dos patrocinadores parceiros.

Uma organização, ao tornar-se patrocinadora de um clube de futebol, insere-se em um contexto estratégico que a torna conhecida até mesmo em âmbito global, via disseminação de sua imagem e o faz acreditando que por meio do fanatismo pelo time os torcedores estarão mais propensos a consumir marcas envolvidas com os respectivos clubes (Meenaghan, 2001; Benazzi \& Borges, 2009).

A relevância do estudo recai sobre as implicações práticas de se demonstrar se os clubes de futebol vêm utilizando as redes sociais de forma efetiva para captação de receitas. Essa premissa vem pautada nos achados de Guerra (2014), o qual buscou identificar a situação de empresas portuguesas quanto as redes sociais e constatou uma facilidade maior na divulgação de produtos e melhoria de imagem da marca em decorrência da maior exposição.

A análise das redes sociais se concentra no Ranking Digital, elaborado pela empresa Ibope Repucom, bem como nas demonstrações financeiras disponibilizadas pelos clubes de futebol nos períodos de 2016 e 2017.

A partir dos achados deste estudo, os gestores de clubes de futebol poderão definir novas estratégias a fim de aumentar sua arrecadação com receitas de patrocínio, bem como tornar suas redes sociais mais atrativas para angariar novos seguidores.

Como contribuição adicional, busca-se verificar qual tipo de rede social (Facebook, Instagram, Twitter e Youtube) possui maior associação com a captação de recursos de patrocínios e publicidade. Considerando que diferentes redes sociais podem apresentar diferentes perfis de público, determinadas empresas podem dar preferência de investimento a clubes com maior presença em determinada rede social.

Em suma, os achados podem auxiliar os gestores dos clubes de futebol na estruturação e gestão de suas mídias sociais, bem como contribuir para maximizar os recursos provenientes de patrocínios e publicidade. 
Dessa forma, observa-se a relevância desse tema no cenário dos clubes. Destaca-se, que não foram encontrados na literatura estudos que promovam a união da análise das receitas de patrocínio e publicidade com o poder de engajamento das redes sociais em clubes de futebol, sendo esta uma possível lacuna a ser preenchida a partir desse estudo.

\section{REFERENCIAL TEÓRICO}

\subsection{Teoria dos Stakeholders}

O termo stakeholders pode ser definido como indivíduo(s) ou grupo(s) que podem impactar e, ao mesmo tempo, são impactados pelas atitudes de determinada empresa. Basicamente, são os personagens envolvidos dentro do contexto da organização (Freeman, 1984). Segundo Silveira, Yoshinaga e Borba (2005), outra definição para stakeholders refere-se aos atores, como por exemplo, empregados, acionistas, clientes e fornecedores que possuem expectativas e interesses em relação à determinada organização, possibilitando a viabilidade da mesma a partir de sua existência.

Dentro desse contexto, surge a teoria dos stakeholders. Donaldson e Preston (1995) destacam que essa teoria busca justamente promover toda a gestão dos stakeholders, trazendo para o centro da questão a forma como serão tomadas as decisões, buscando analisar todos os envolvidos, dentro de um ambiente com múltiplos propósitos. Cabe salientar que, de acordo com Phillips, Freeman e Wicks (2003), o objetivo nessa teoria é o tratamento de todas as partes de maneira igualitária, que permita a maximização do interesse comum em prol da organização.

Nesse sentido, é crucial o entendimento acerca da expectativa de cada stakeholder, a fim de implementar estratégias que possibilitem alinhar os interesses dos envolvidos dentro de um mesmo cenário (Clarkson, 1995). Roberto e Serrano (2007) destacam que, ao buscar a maximização de receitas, a organização deve obter um relacionamento mais efetivo com seus stakeholders, a fim de angariar mais recursos com a satisfação de seus clientes.

Complementando essa visão, Freeman e Philips (2002) relatam que é a partir da elaboração de estratégias para promover o gerenciamento com os stakeholders que a organização deve pautar suas ações, a fim de promover seu desempenho.

Com o intuito de analisar situações a partir dessa teoria, ressalta-se que existem três abordagens possíveis, sendo elas: normativa, instrumental e descritiva. O conceito normativo trata da função da empresa bem como um dos valores morais balizadores da tomada de decisão, o instrumental foca em objetivos de comportamento de desempenho financeiro na gestão dos stakeholders e a abordagem descritiva utiliza-se da teoria para o entendimento dos aspectos e ações adotados pela organização (Donaldson \& Preston, 1995).

Diante disso, é possível traçar o cenário dos principais stakeholders analisados no presente estudo com os seguintes participantes: as empresas patrocinadoras, os clubes de futebol e o público atuante nas redes sociais. Isso é visto mais detalhadamente no tópico a seguir.

\subsubsection{Teoria dos Stakeholders no contexto dos clubes de futebol}

Trazendo a discussão para o cenário dos clubes de futebol, pode-se dizer que existem diferentes stakeholders que podem impactar o processo de gestão dos clubes e que devem ser considerados pelos dirigentes no processo de tomada de decisão. Outro ponto a destacar refere-se à dificuldade dos clubes no processo de avaliação da relevância de cada stakeholder por parte de seus gestores, a fim de possibilitar uma gestão mais profissional do setor desportivo (Siqueira, Pajanian \& Telles, 2015)

Ressalta-se que o contexto que envolve os clubes é diferenciado do mercado tradicional e possui pontos específicos de análise. Nesse caso, o objetivo dos clubes é o seu desempenho dentro dos campeonatos em que participam, buscando, paralelamente, sua viabilidade econômica (Hamil, Michie, Oughton \& Warby, 2000).

Dentre os stakeholders existentes há a figura dos atletas, que a partir de seu desempenho e comercialização constituem grande parte desse cenário e também dos patrocinadores, que por meio de seus contratos buscam alavancar suas marcas e são uma fonte de recursos relevantes para os clubes (Costa \& Silva, 2009).

Outra parte integrante desse sistema são os torcedores, onde é fundamental que os clubes se utilizem desses stakeholders para obtenção de receitas e criação de relações interempresariais que busquem maior proximidade (Leoncini \& Silva, 2004). Uma das formas que possibilita esse aprofundamento entre os stakeholders e o clube é por meio das redes sociais, conforme discutido na próxima seção.

\subsection{Redes Sociais}

Com relação às redes sociais, as pesquisas nessa área surgem em um contexto de aumento da utilização de tecnologias da informação e da conscientização sobre a relevância dos ativos da organização, buscando-se analisar o papel dessas mídias como ferramenta de divulgação de informações.

O estudo das redes sociais permite um conhecimento sobre as relações de poder hierárquicas e espontâneas de uma organização, buscando compreender de que forma a dinâmica do conhecimento interfere nesse processo (Marteleto, 2001).

Essas relações podem ser avaliadas por meio de diversas métricas, sendo importante citar: métricas de visibilidade (número de visualizações, curtidas, etc.); métricas de respeito (número de avaliações recebidas, seguidores nas redes 
sociais, etc.) e; métricas de sentimento (análise de conteúdos textuais e informações subjetivas) (Sterne, 2010). Por meio de cada uma delas, diferentes estratégias podem ser elaboradas com base no objetivo da organização.

Dessa forma, a comunicação nas redes sociais amplia a esfera de influência do marketing, possibilitando que usuários criem e compartilhem informações significativas entre empresas e seus clientes (Hanna, Rohm \& Crittenden, 2011). É possível, portanto, a criação e troca de conteúdo, permitindo a conexão entre empresas com seus atuais clientes e clientes em potenciais, oferecendo novas oportunidades de pesquisa, facilitando o processo de comunicação e atingindo diversos usuários (Kaplan \& Haenlein, 2010; Safko \& Brake, 2009; Lee, Hutton \& Shu, 2015).

Corroborando com isso, Mangold e Faulds (2009) destacam que a partir das redes sociais o processo de comunicação e interação foi modificado, tornando-se uma importante ferramenta onde os clientes buscam maiores informações e avaliações de produtos antes da decisão final de compra.

Tendo conhecimento disso, muitos gestores têm se atentado para a análise de estratégias de utilização de redes sociais para o alcance de seus objetivos organizacionais e aumento de suas vendas, contratando inclusive gerentes de mídia social para o desenvolvimento desses canais online (Molina, 2014; Deloitte, 2016). Nesse sentido, os clubes usam as redes sociais para se comunicar com os torcedores, permitindo um contato direto com os mesmos e, por meio dessa relação, buscam estabelecer retornos econômicos (Sšderman \& Dolles, 2013; Rosen, 1981).

A popularidade das redes sociais contribui para o desenvolvimento do valor da marca de clubes de futebol, além de permitir infinitas possibilidades por meio da oferta de novos canais de distribuição em várias plataformas digitais (Boyle \& Haynes, 2004).

A partir desse entendimento é possível traçar o seguinte cenário: empresas patrocinam os clubes com o intuito de expor sua marca; os clubes, a fim de continuar ganhando com esse tipo de receita, postam em suas redes sociais imagens, vídeos e textos destacando seus patrocinadores e, com isso, estreitam o relacionamento com seus torcedores e divulgam as marcas; os seguidores das redes sociais buscam informações dos clubes e, nesse contexto, acabam conhecendo as organizações patrocinadoras.

Sendo assim, o envolvimento da organização com as redes sociais também deve ser considerado por meio do impacto em seu desempenho, já que na atualidade há uma necessidade constante por informações que podem ser divulgadas de maneira efetiva (Rubin \& Rubin, 2010). Ou seja, a relação da popularidade dos clubes nas redes sociais onde possuem perfil permite a disseminação do conteúdo dos produtos oferecidos pelos patrocinadores e pode contribuir tanto para o clube quanto para as marcas que investem nele.

Um ponto relevante é que, de acordo com Sterne (2010), ao analisar o contexto de redes sociais, deve-se buscar pelo menos um dos seguintes objetivos: maximização de receitas, redução de custos ou aumento da satisfação do consumidor.

Cabe ressaltar que o presente estudo está em linha com Sterne (2010), já que busca verificar qual a relação entre a quantidade de seguidores nas redes sociais (relatada como uma métrica de respeito) e a captação de receitas de patrocínio.

\subsection{Estudos Anteriores Relacionados ao Tema}

Nesta seção são relatados alguns estudos que tratam do objeto de estudo, expondo seus principais achados a fim de servir de suporte para a escolha das variáveis que foram utilizadas no modelo empírico.

Lardo, Dumay e Russo (2017) estudaram essa relação em 20 clubes listados na Europa, juntamente com outras variáveis como despesa e valor de mercado, tendo obtido como resultado a relação positiva entre a popularidade dos clubes nas redes sociais e as receitas auferidas por eles.

Dantas, Machado e Macedo (2015) analisaram o patamar de eficiência dos clubes e verificaram significância desta com a receita e o desempenho esportivo. Acreditam que o desempenho do clube nas competições participantes interfere na em seu faturamento anual.

O estudo de Albuquerque, Silva e Maluf (2014) teve como objetivo destacar as relações entre o faturamento de empresas siderúrgicas com variáveis macroeconômicas e obtendo como resultado a evidenciação de que a utilização do PIB defasado tem influência na receita do ano posterior. Isso pode ocorrer em outros setores, tais como o esportivo, sendo relevante uma análise com essa abordagem.

Paniagua e Sapena (2014) buscaram analisar a influência das redes sociais no valor das ações das empresas espanholas listadas no IBEX (índice com as empresas de maior cotação), pois as consideravam nova nas redes sociais. Como resultado, foi verificado a existência de relação positiva entre as variáveis, sendo ainda destacado que para isso a empresa necessita atingir uma quantidade expressiva de seguidores.

\section{METODOLOGIA}

Trata-se de um estudo explicativo e quantitativo cujos documentos utilizados são as demonstrações financeiras dos clubes bem como o ranking digital elaborado pelo Ibope Repucom. O período de análise compreende os anos de 2016 e 2017, pois este é o período com dados disponíveis no ranking.

Esse ranking é elaborado a partir dos quarenta clubes com maior número de seguidores e traz as informações das seguintes redes sociais: Facebook, Twitter, Instagram e Youtube. Tendo em vista a disponibilidade de obter os dados do ranking nesses dois anos supracitados, a amostra é não probabilística por conveniência e está composta por 32 dos 
clubes componentes do ranking digital que divulgaram suas demonstrações nos dois anos requeridos, sendo tabulados de forma manual.

As variáveis de interesse referem-se à receita de patrocínio e publicidade dos clubes de futebol e a presença de seguidores nas redes sociais visando avaliar sua relação a partir do engajamento dos clubes nas redes existentes. Paniagua e Sapena (2014) afirmam que a partir da utilização das redes sociais a organização pode ser afetada em diversas áreas, tanto no relacionamento com os indivíduos que acompanham os perfis por meio destas redes como na obtenção de receitas a partir da exposição de marcas interessadas. A intenção no presente estudo é justamente essa, a de verificar o impacto dos seguidores dessas redes na receita de patrocínio dos clubes.

Como variáveis de controle, utilizou-se o desempenho esportivo divulgado a partir da pontuação no ranking da Confederação Brasileira de Futebol (CBF) que contempla todos os clubes de futebol profissional brasileiro), o tamanho do clube mensurado com base no ativo total em termos logarítmicos e o produto interno bruto (PIB) de cada estado ao qual o clube pertence, sendo esta última uma variável macroeconômica e utilizada de forma defasada, ou seja, a partir de seus valores no ano anterior, no pressuposto de que estes impactam nos investimentos do clube do ano posterior.

Como Silva e Silva (2015) destacam que o PIB faz parte do contexto esportivo, pois cerca de $2 \%$ de seu total é decorrente das transações que ocorrem nesse setor, buscou-se utilizá-lo como variável de controle, a fim de testar se este influencia na receita analisada no presente estudo.

Ou seja, acredita-se nos seguintes pressupostos: a quantidade de seguidores nas redes sociais impacta na receita de patrocínio, pois permite a exposição a um maior número de pessoas; clubes maiores tendem a ter receitas maiores; o PIB influencia na disponibilidade de recursos a serem alocados pelos patrocinadores e o desempenho esportivo promove uma maior expectativa de exposição do clube, elevando assim a receita de patrocínio e publicidade.

Com base no exposto e fundamentado a partir de estudos anteriores (Lardo, Dumay \& Russo, 2017; Dantas, Machado \& Macedo, 2015; Albuquerque, Silva \& Maluf, 2014; Paniagua \& Sapena, 2014), formula-se a seguinte hipótese de pesquisa:

$\mathrm{H}_{1}$ : Existe relação positiva entre a receita de patrocínio e publicidade e o número de seguidores dos perfis dos clubes nas redes sociais.

Quanto ao modelo econométrico adotado, optou-se pela regressão de dados em painel, compondo um painel balanceado com 64 observações. Gujarati (2006, p. 525) afirma que "ao estudar observações de corte transversal repetidas, os dados em painel são mais indicados para estudar a dinâmica da mudança”. Para a escolha do modelo de painel adequado, utilizaram-se os testes de Chow, que avalia o melhor entre pooled e efeito fixo; teste de Hausman, melhor modelo entre efeito fixo e efeito aleatório e o teste de Breuch-Pagan, que avalia o melhor modelo entre pooled e efeito aleatório (Fávero, Belfiore, Silva \& Chan, 2009).

O teste de Chow apresentou p-valor de 0,0000, indicando efeito fixo; o teste de Hausmann apresentou p-valor de 0,9616, indicando efeito aleatório e, por fim, o teste de Breuch-Pagan validou o efeito aleatório no estudo, já que apresentou p-valor de 0,0000 .

Sendo assim, apresenta-se o modelo econométrico utilizado.

RECPAT $=\alpha_{0}+\alpha_{1}$ REDSOC $_{i, t}+\alpha_{2} \operatorname{DESESP}_{i, t}+\alpha_{3} \mathrm{TAM}_{i, t}+\alpha_{4} \mathrm{PIB}_{i, t-1}+\alpha_{5} \mathrm{ANO}_{i, t}+\varepsilon_{i, t}$

Onde:

RECPAT é a receita de patrocínio e publicidade do clube (em termos logarítmicos).

REDSOC é a presença nas redes sociais, obtido pela soma dos usuários das redes sociais dos clubes estudados (em termos logarítmicos).

DESESP é o indicador de desempenho esportivo, obtido pelo ranking da CBF.

TAM é o tamanho do clube mensurado pelo Ativo Total em termos logarítmicos.

PIB é a o valor do Produto Interno Bruto do Estado que o clube pertence em t-1.

ANO é a dummy de ano, que possui valor 1 caso pertença ao ano de 2016 e 0 caso pertença a 2017.

$\varepsilon$ é o termo de erro.

t é o período da análise.

Para ampliar a análise, apuraram-se resultados levando-se em consideração os coeficientes específicos de cada rede social estudada em termos logarítmicos, de forma a verificar se alguma rede social específica pode influenciar mais os investimentos em patrocínios e publicidade, conforme apresentado a seguir. 


$$
\begin{aligned}
& \operatorname{RECPAT}_{i, t}=\alpha_{0}+\alpha_{1} \mathrm{INST}_{i, t}++\alpha_{2} \mathrm{DESESP}_{i, t}+\alpha_{3} \mathrm{TAM}_{i, t}+\alpha_{4} \mathrm{PIB}_{i, t-1}+\alpha_{5} \mathrm{ANO}_{i, t}+\varepsilon_{i, t} \\
& \operatorname{RECPAT}_{i, t}=\alpha_{0}+\alpha_{1} \mathrm{FACEB}_{i, t}++\alpha_{2} \mathrm{DESESP}_{i, t}+\alpha_{3} \mathrm{TAM}_{i, t}+\alpha_{4} \mathrm{PIB}_{i, t-1}+\alpha_{5} \mathrm{ANO}_{i, t}+\varepsilon_{i, t} \\
& \operatorname{RECPAT}_{i, t}=\alpha_{0}+\alpha_{1} \mathrm{YOUT}_{i, t}++\alpha_{2} \mathrm{DESESP}_{i, t}+\alpha_{3} \mathrm{TAM}_{i, t}+\alpha_{4} \mathrm{PIB}_{i, t-1}+\alpha_{5} \mathrm{ANO}_{i, t}+\varepsilon_{i, t} \\
& \operatorname{RECPAT}_{i, t}=\alpha_{0}+\alpha_{1} \mathrm{TWIT}_{i, t}++\alpha_{2} \mathrm{DESESP}_{i, t}+\alpha_{3} \mathrm{TAM}_{i, t}+\alpha_{4} \mathrm{PIB}_{i, t-1}+\alpha_{5} \mathrm{ANO}_{i, t}+\varepsilon_{i, t}
\end{aligned}
$$

Onde:

INST é o número de seguidores do clube no Instagram.

FACEB é o número de curtidas no perfil do clube no Facebook.

YOUT é o número de inscritos no perfil do clube no Youtube.

TWIT é o número de seguidores no perfil do clube Twitter.

Tendo em vista a hipótese de pesquisa, espera-se que o coeficiente de interesse das regressões ( sejam positivos e estatisticamente significativos. Em sendo assim, nos casos específicos, analisa-se a magnitude dos coeficientes com vistas a identificar a rede social com maior influência.

As limitações da pesquisa referem-se ao reduzido número de observações, que decorre da ferramenta utilizada para verificação do impacto nas redes sociais, bem como a restrição dos resultados somente aos clubes analisados, não se permitindo generalização dos mesmos.

\section{ANÁLISE DOS RESULTADOS}

Primeiramente, apresenta-se a estatística descritiva referente às variáveis do estudo, conforme Tabela 1.

Tabela 1 - Estatística Descritiva

\begin{tabular}{c|c|c|c|c|}
\hline Variáveis & Média & Desvio Padrão & Mínimo & Máximo \\
\hline $\begin{array}{c}\text { RECPAT } \\
\text { (R\$ mil) } \\
\text { REDSOC } \\
\text { (Qtde) }\end{array}$ & 18.450 & 26.460 & 236,1 & 130.900 \\
$\begin{array}{c}\text { DESESP } \\
\text { (Pontos) }\end{array}$ & 3.681 .000 & 4.716 .000 & 168.500 & 19.580 .000 \\
$\begin{array}{c}\text { TAM } \\
\text { (R\$ mil) }\end{array}$ & 8.997 & 3.746 & 2.537 & 1.331 .000 \\
PIB \\
(R\$ mil)
\end{tabular}

Fonte: Dados da Pesquisa (2019).

Nota: RECPAT: receita de patrocínio e publicidade; REDSOC: rede social; DESESP: desempenho esportivo; TAM: tamanho; e PIB: produto interno bruto.

A partir da análise da estatística descritiva é possível observar o porquê de serem utilizadas as variáveis (exceto a variável DESESP) em termos logarítmicos, ou seja, em função do elevado desvio padrão. Outro ponto refere-se aos valores mínimos e máximos observados já que na amostra há clubes das séries $\mathrm{A}, \mathrm{B}$ e $\mathrm{C}$ e que possuem diferença expressiva, tanto em termos de arrecadação quanto de visibilidade nas redes sociais.

Tratando-se da correlação entre as variáveis, as mesmas podem ser observadas na Tabela 2. 
Tabela 2 - Matriz de Correlação

\begin{tabular}{c|c|c|c|c|c}
\hline Variáveis & RPAT & REDSOC & DESESP & TAM & PIB \\
\hline RECPAT & 1 & & & \\
REDSOC & $0,8 * *$ & 1 & & \\
DESESP & $0,8 * *$ & $0,8 * *$ & 1 & \\
TAM & $0,7^{* *}$ & $0,6 * *$ & $0,7 * *$ & 1 & \\
PIB & $0,4 * *$ & $0,4 * *$ & $0,5 * *$ & $0,2 *$ & 1 \\
\hline
\end{tabular}

Fonte: Dados da Pesquisa (2019).

Nota: Os asteriscos mostram o nível de significância das variáveis explicativas sendo, ** significativa no nível 0,05 e * correlação significativa no nível 0,1. RECPAT: receita de patrocínio e publicidade; REDSOC: rede social; DESESP: desempenho esportivo; TAM: tamanho; PIB: produto interno bruto; e ANO: dummy de ano.

Ressalta-se que todas as variáveis apresentaram correlação estatisticamente significativas entre si, sendo a significância a um nível de 5\% entre todas as variáveis, exceto TAM e PIB que apresentaram correlação significativa ao nível de $10 \%$. Esse cenário pode sugerir que clubes que possuem maior receita de patrocínio e publicidade estão associados ao maior número de seguidores nas redes sociais, ao bom desempenho nos campeonatos que disputam, ao seu tamanho (mensurado em seu ativo) bem como ao PIB do Estado onde estão localizados.

Pautando-se na premissa de Vincent e Weir (2012) de que o limite aceitável entre as variáveis independentes é de correlações até 0,8 , as variáveis do modelo enquadram-se dentro do estabelecido, não prejudicando o andamento do estudo.

Contudo, a fim de verificar se havia algum problema de autocorrelação de resíduos entre as variáveis, foi feito o teste de Durbin Watson, que obteve p-valor 0,3892, demonstrando que não há problema de autocorrelação dos resíduos.

Quanto aos resultados obtidos na regressão, estes são apresentados na Tabela 3. Inicialmente, destaca-se que foram feitos os testes de diagnóstico de painel e por meio dos resultados dos testes de Breusch-Pagan e Hausman, foi utilizada a abordagem por efeitos aleatórios.

Tabela 3 - Resultados da Regressão - Modelo Completo

\begin{tabular}{|c|c|c|c|}
\hline \multicolumn{2}{|c|}{ Variável Explicativa ou Independente } & \multicolumn{2}{|c|}{ Coeficiente } \\
\hline \multicolumn{2}{|c|}{ REDSOC } & \multicolumn{2}{|c|}{$\begin{array}{c}0,5805^{\star \star \star} \\
(1,1525)\end{array}$} \\
\hline \multicolumn{2}{|c|}{ DESESP } & \multicolumn{2}{|c|}{$\begin{array}{c}0,0000 \\
(1,1871)\end{array}$} \\
\hline \multicolumn{2}{|c|}{ TAM } & \multicolumn{2}{|c|}{$\begin{array}{c}0,1615^{\star} \\
(1,6851)\end{array}$} \\
\hline \multicolumn{2}{|c|}{ PIB } & \multicolumn{2}{|c|}{$\begin{array}{c}0,0273 \\
(0,4753)\end{array}$} \\
\hline \multicolumn{2}{|c|}{ ANO } & \multicolumn{2}{|c|}{$\begin{array}{c}-0,0611 \\
(-1,1665)\end{array}$} \\
\hline \multicolumn{2}{|c|}{ Constante } & \multicolumn{2}{|c|}{$\begin{array}{c}1,4109 \\
(1,1525)\end{array}$} \\
\hline \multicolumn{4}{|c|}{ Informações Adicionais } \\
\hline $\mathrm{R}^{2}(\%)$ & 66,55 & $\mathrm{R}^{2}$ Ajustado (\%) & 63,69 \\
\hline F (estat) & 23,10 & $\mathrm{~F}$ (p-value) & 0,0000 \\
\hline
\end{tabular}

Fonte: Dados da Pesquisa (2019).

Nota: Estatística T reportada entre parênteses; os asteriscos mostram o nível de significância das variáveis explicativas sendo, *** significativa no nível 0,01 e * significativa no nível 0,10. RECPAT: receita de patrocínio e publicidade; REDSOC: rede social; DESESP: desempenho esportivo; TAM: tamanho; PIB: produto interno bruto; e ANO: dummy de ano. 
Quanto aos testes para os pressupostos da regressão, o teste de White obteve p-valor de 0,7291 sinalizando que não há problema de heterocedasticidade. Já o teste de normalidade apresentou p-valor de 0,0001, o que indica que os dados não seguem uma distribuição normal. Nesse caso, houve relaxamento do pressuposto em decorrência do teorema do limite central. Em relação à multicolinearidade, o valor FIV de todas as variáveis ficou entre 1,023 e 5,002, não sinalizando problema entre as variáveis.

A regressão como um todo se mostrou significativa, conforme a Tabela 3, já que apresentou p-valor de 0,0000, ou seja, menor que o nível de significância de $1 \%$. O $\mathrm{R}^{2}$ relata que $66,55 \%$ das receitas de patrocínio e publicidade podem ser explicadas pelas variáveis independentes utilizadas no modelo.

É possível observar que a variável rede social se mostrou significativa e positiva ao nível de 1\%, corroborando, assim, com a hipótese do estudo de que há relação entre as receitas de patrocínio e publicidade dos clubes e s a quantidade de seguidores nas redes sociais. O resultado vai ao encontro com estudos que destacam que a utilização de redes sociais por parte das organizações traz como uma das vantagens o aumento na receita (Gilmore, Gallagher \& Henry, 2007; Paniagua \& Sapena, 2014; Lardo, Dumay \& Russo, 2017).

Esse achado também está em linha com o estudo de Scharf (2010), que verificou que o patrocinador busca reconhecimento no momento em que decide fazer essa parceria com um clube de futebol, sendo que as redes sociais são um meio efetivo de propagação de imagem, trazendo para marca uma posição de destaque.

Outra variável que se mostrou positiva e significativa, mas a um nível de significância de 10\%, foi à relacionada a tamanho, demonstrando que clubes com ativos maiores possuem maior visibilidade perante as organizações, são mais procurados para patrocínio e possuem as maiores receitas. Estender (2013) relatou esse aspecto ao afirmar que os patrocinadores estão em busca de clubes que possuem grande magnitude, pois tem maior destaque perante os stakeholders, acarretando uma maior exposição.

A variável ano não se mostrou significativa, sugerindo que não houve impacto nas receitas dos clubes no que tange ao ano da arrecadação. Isso contrasta com a análise feita pelo Sports Value (2018) que destacou aumento em $27 \%$ no crescimento das receitas de patrocínio nesse período.

O PIB, diferente do esperado, não apresentou significância, contrastando com o observado por Albuquerque, Silva e Maluf (2014). Pode-se justificar isso na possibilidade de o setor esportivo não ser exposto tanto a questão macroeconômica do país e sim com as negociações feitas em determinado ano.

Cabe destacar que cada uma das redes sociais tem suas peculiaridades e públicos, sendo o Facebook, a rede de maior destaque; o Twitter, canal criado para melhoria de comunicação e usado pelas organizações para promover até mesmo pesquisas; e o Youtube, para divulgação de vídeos (Safko \& Brake, 2009). Já o Instagram foi criado com o intuito de compartilhamento de fotos, onde usuários podem curtir, comentar e ainda compartilhar essas fotos nas outras redes sociais $(\mathrm{G} 1,2012)$.

A fim de obter considerações acerca do poder explicativo de cada uma das redes sociais de forma separada, buscou-se avaliar por meio da regressão o coeficiente e significância de cada uma. Essa análise permite verificar qual delas possui maior relação com as receitas de patrocínio e publicidade.

Destaca-se que os resultados estão expostos da seguinte forma: no modelo (1), utilizou-se somente o Instagram como rede social; no modelo (2), utilizou-se o Facebook; no modelo (3) o Youtube e no modelo (4) o Twitter. Os resultados são apresentados na Tabela 4.

Tabela 4 - Resultados da Regressão - Modelo Decomposto

\begin{tabular}{|c|c|c|c|c|}
\hline $\begin{array}{l}\text { Variável Explicativa ou } \\
\text { Independente }\end{array}$ & (1) & (2) & (3) & (4) \\
\hline INST & $\begin{array}{c}0,4718^{\star \star \star} \\
(3,491)\end{array}$ & - & - & - \\
\hline FACEB & - & $\begin{array}{c}0,4676^{\star \star \star} \\
(3,150)\end{array}$ & - & - \\
\hline YOUT & - & - & $\begin{array}{l}0,0376 \\
(1,255)\end{array}$ & - \\
\hline TWIT & - & - & - & $\begin{array}{c}0,4601^{\star * *} \\
(3,103)\end{array}$ \\
\hline DESESP & $\begin{array}{c}0,00004^{*} \\
(1,991)\end{array}$ & $\begin{array}{c}0,1632^{\star} \\
(1,770)\end{array}$ & $\begin{array}{c}0,00008^{\star \star \star *} \\
(3,774)\end{array}$ & $\begin{array}{c}0,00003 \\
(1,352)\end{array}$ \\
\hline TAM & $\begin{array}{l}0,1355 \\
(1,352)\end{array}$ & $\begin{array}{l}0,1632 \\
(1,642)\end{array}$ & $\begin{array}{c}0,2011^{*} \\
(1,968)\end{array}$ & $\begin{array}{l}0,1814^{\star} \\
(1,838)\end{array}$ \\
\hline PIB & $\begin{array}{c}0,02989 \\
(0,556)\end{array}$ & $\begin{array}{l}0,0219 \\
(0,386)\end{array}$ & $\begin{array}{l}0,0251 \\
(0,428)\end{array}$ & $\begin{array}{l}0,0267 \\
(0,460)\end{array}$ \\
\hline
\end{tabular}




\begin{tabular}{|c|c|c|c|c|}
\hline $\begin{array}{l}\text { Variável Explicativa ou } \\
\text { Independente }\end{array}$ & (1) & (2) & (3) & (4) \\
\hline ANO & $\begin{array}{c}-0,0412 \\
(-1,1334)\end{array}$ & $\begin{array}{l}-0,089 * * \\
(-2,599)\end{array}$ & $\begin{array}{c}-0,0884^{\star \star} \\
(-2,341)\end{array}$ & $\begin{array}{l}-0,0463 \\
(-1,165)\end{array}$ \\
\hline Constante & $\begin{array}{c}2,6177^{\star *} \\
(2,507)\end{array}$ & $\begin{array}{l}2,1951^{*} \\
(1,885)\end{array}$ & $\begin{array}{c}4,1497^{\star \star \star} \\
(4,124)\end{array}$ & $\begin{array}{r}2,1776^{\star} \\
(1,842)\end{array}$ \\
\hline \multicolumn{5}{|c|}{ Informações Adicionais } \\
\hline $\mathrm{R}^{2}(\%)$ & 63,00 & 63,24 & 57,69 & 63,71 \\
\hline $\mathrm{R}^{2}$ Ajustado (\%) & 59,81 & 60,07 & 54,05 & 60,59 \\
\hline F (p-value) & 0,0000 & 0,0000 & 0,0000 & 0,0000 \\
\hline
\end{tabular}

Fonte: Dados da Pesquisa (2019).

Nota: Estatística T reportada entre parênteses; os asteriscos mostram o nível de significância das variáveis explicativas sendo, *** significativa no nível 0,01, ** significativa no nível 0,05 e * significativa no nível 0,10. INST: instagram; FACEB: facebook; YOUT: youtube; TWIT: twitter; DESESP: desempenho esportivo; TAM: tamanho; PIB: produto interno bruto; e ANO: dummy de ano.

Pode-se observar que todas as regressões do modelo decomposto mostraram-se significativas. O R ${ }^{2}$ destes modelos ficou em torno de $60 \%$, sugerindo que não há grandes mudanças no poder explicativo das variáveis independentes quando as redes sociais são segregadas. Outro ponto a observar é que, com exceção do Youtube, todas as demais redes sociais se mostraram significativas quando rodadas na regressão decomposta, com significância ao nível de $1 \%$.

Diferente do observado na regressão com as redes sociais conjuntas, o desempenho esportivo foi significativo no modelo utilizando a rede Instagram, Facebook e Twitter. Isso pode ser devido ao fato de que um clube com desempenho melhor faz com que seus stakeholders queiram estar em contato direto com as fotos, notícias e comentários publicados nessas redes e isso impacta nas receitas de patrocínio e publicidade. Essa relação positiva de receita e desempenho esportivo corrobora com o achado de Dantas, Machado e Macedo (2015).

Nesse contexto, Dantas e Boente (2011), relatam um ciclo: clubes com desempenho favorável possuem maior captação de receita e com isso possuem verba para contratação de bons jogadores, o que os faz terem um bom desempenho esportivo.

Já a variável ano mostrou-se significativa quando utilizadas nos modelos com as variáveis relacionadas ao Facebook e Twitter, demonstrando um destaque nesses modelos no ano de 2016.

Outro contraste observado é que no estudo de Paniagua e Sapena (2014), observou-se que o Twitter era a rede social com maior pode para impactar o desempenho, quando comparado ao Facebook. Já no presente estudo o modelo com Facebook apresentou coeficiente e $\mathrm{R}^{2}$ levemente superior ao Twitter, demonstrando que no setor esportivo, em âmbito brasileiro, esta rede social está em destaque.

Respaldando essa questão, pode-se citar o estudo de Mokfianski e Alvetti (2017), que aponta sobre a análise do uso do Facebook pelo Coritiba Football Club e verificaram que esta rede social está sendo amplamente utilizada pelo clube, aprimorando a comunicação com os torcedores. Nesse sentido, esta rede poderia ser usada de forma estratégica de angariar mais patrocínio e permitir com que o clube planeje de que forma irá expor essas marcas.

Ainda assim, os resultados demonstraram que não há grandes diferenças entre o poder explicativo delas, sendo importante para os clubes de futebol estar presente em todas as redes sociais, pois de forma conjunta há uma maximização de visibilidade, já que o maior $\mathrm{R}^{2}$ observado do estudo foi visto na regressão combinada (Tabela 3) e isso é um grande atrativo para os patrocinadores.

\section{CONSIDERAÇÕES FINAIS}

Esse estudo buscou verificar a relação entre a presença de torcedores nas redes sociais e a captação de recursos de patrocínio e publicidade. Foram analisados 32 clubes nos anos de 2016 e 2017 que disponibilizaram suas demonstrações contábeis e constavam na análise de rankings digitais elaborada pelo Ibope Repucom.

Barros, Scafarto e Samagaio (2014) afirmam que a receita de patrocínio é uma fonte diferenciada de captação de recursos e demanda uma estratégia de comunicação bem definida para satisfazer os patrocinadores.

Uma forma de os clubes de futebol promoverem a comunicação entre stakeholders na atualidade é por meio das redes sociais, sendo um canal que possibilita estratégias institucionais, onde os torcedores e até mesmo patrocinadores podem relacionar-se (Mokfianski \& Alvetti, 2017). Nesse sentido, a análise da relação entre o número de seguidores dos perfis dos clubes nas redes sociais e sua receita de patrocínio e publicidade permite observar se esta ferramenta tem sido utilizada para pautar uma organização em seu processo de escolha no momento em que decide tornar-se patrocinadora de um clube.

O patrocínio possibilita uma maior exposição da marca, trazendo benefícios a partir da exposição desta nos campeonatos que o clube disputa. A partir da análise da regressão elaborada, foi possível constatar que há relação positiva 
e significativa entre a receita de patrocínio dos clubes e a quantidade de seguidores nas redes sociais de forma conjunta, sugerindo que essa relação se deve a atenção dos patrocinadores no engajamento dos torcedores perante as redes sociais e levam isso em consideração no momento em que fazem o contrato com os clubes.

Isso ocorre, pois as redes sociais permitiram uma mudança nas estratégias das organizações com o intuito de fortalecer o relacionamento com os consumidores, angariar recursos e aumentar a exposição de marcas e produtos (Sousa, Silva, Pinto, Nascimento \& Rabelo, 2018).

Outra variável que se mostrou significativa nesse cenário foi o tamanho do clube, ou seja, os patrocinadores consideram investir mais em clubes maiores. Isso faz sentido, tendo em vista que estes clubes possuem uma exposição maior nos campeonatos disputados.

Já nas regressões decompostas, estas mostraram cada uma das redes sociais significativas, porém, sem grandes discrepâncias entre o coeficiente obtido em cada uma delas, sugerindo que o melhor cenário é o da inserção dos clubes em todas as redes sociais existentes de forma integrada.

Mullin, Hardy e Sutton (2004) já haviam destacado que a exposição dos clubes em diferentes campeonatos pode ser vista como uma forma de divulgação gratuita dos patrocinadores. Com a disseminação das redes sociais, esta afirmação pode ser expandida para perfis oficiais dos clubes no Facebook, Instagram, Twitter e Youtube.

Salles e Castro Junior (2015) analisaram os clubes cariocas frente às redes sociais e constataram que as estratégias de atratividade a partir delas ainda são muito limitadas, sendo fundamental que sejam reavaliadas a fim de utilizar-se dessa importante ferramenta de promoção de imagem na era atual.

Sendo assim, os clubes devem aprimorar-se em formas de atrair seus torcedores para seus perfis nas redes sociais, permitindo que seus patrocinadores fiquem mais interessados e essa fonte de receita possa elevar-se com a nova realidade de exposição de imagem dos clubes.

Recomenda-se para estudos futuros aumentar o período de análise temporal bem como a amostra, incluindo até mesmo clubes internacionais para verificar se o comportamento se mantém. Outra possibilidade seria a discussão até mesmo das seleções e suas receitas de patrocínio e publicidade com o engajamento nas redes sociais, permitindo a ampliação dessa questão a nível global.

\section{REFERÊNCIAS}

Albuquerque, P. H. M., Silva, L. D. C., \& Maluf, Y. S. (2014). Estimação da influência de variáveis macroeconômicas sobre o faturamento de organizações siderúrgicas usando o ARMAX. Gestão \& Produção, 21(3), p. 648-659,

Barros, C., Scafarto, V., \& Samagaio, A. (2014). Cost performance of Italian football clubs: Analysingthe role of marketing and sponsorship. International Journal of Sports Marketing and Sponsorship, 15(4), p. 59-77.

Benazzi, J. R. S.; \& Borges C. N. (2009). Emoção, exposição e vendas: Análise do patrocínio realizado por marcas de artigos esportivos no futebol brasileiro. Anais do Simpósio de Excelência em Gestão e Tecnologia. Rio de Janeiro, RJ, Brasil.

Boyle, R.; \& Haynes, R. (2004). Football in the new media age. London: Routledge.

Brogan, Chris. (2012). ABC das Midias Sociais. São Paulo: Brumo.

Cardia, Wesley. (2004). Marketing e patrocínio esportivo. Editora Bookman.

Clarkson, M. E. (1995). A stakeholder framework for analyzing and evaluating corporate social performance. Academy of Management Review, 20(1), p. 92-117.

Costa, C. E. S.; \& Silva, R. C. (2006). Empresarização e controle organizacional: um estudo nos clubes de futebol em Santa Catarina. Cadernos Ebape Br- FGV, 4(4), p.1-16.

Dantas, M. G. S., Machado, M. A. V., \& Macedo, M. A. da S. (2015). Fatores determinantes da eficiência dos clubes de futebol do Brasil. Advances in Scientific and Applied Accounting, 8(1), p. 113-132.

Deloitte. (2016). Top of the table - football money league. Sport Business Group, p. 1-36.

Donaldson, T.; \& Preston, L. E. (1995). The stakeholder theory of the corporation: concepts, evidence and implications. Academy of Management Review, 20, p. 65-91.

Estender, A. C. (2013). A Importância da administração profissional para os clubes de futebol. Revista Administração em Diálogo, 15(3), 18-32.

Fávero, L. P.; Belfiore, P. P.; Silva, F. L. da; \& Chan, B. L. (2009). Análise de dados: modelagem multivariada para tomada de decisões. Rio de Janeiro: Elsevier.

Freeman, R. E. (1984). Strategic management: A stakeholder approach. Boston: Pitman.

G1. (2012). Entenda a curta história do Instagram, comprado pelo Facebook. 2012. Recuperado em 20 janeiro, 2019, de http:// g1.globo.com/tecnologia/noticia/2012/04/entenda-curta-historia-do-instagram-comprado-pelo-facebook.html.

Gilmore, A., Gallagher, D., \& Henry, S. (2007). E-marketing and SMEs: operational lessons for the future. European Business Review, 19(3), 234-247.

Guerra, N. J. M. (2014). A adoção e implementação das aplicações de social media: as grandes empresas portuguesas. Tese de Doutorado em Gestão, Universidade de Évora, Évora, Portugal. 
Gujarati, Damodar N. (2006). Econometria básica. Rio de Janeiro: Elsevier.

Hamil, S.; Michie, J.; Oughton, C.; \& Warby, S. (2000). Recent developments in football ownership. Soccer \& Society, 1(3), p. 1-10.

Hanna, R.; Rohm, A.; \& Crittenden, V. L. (2011). We're all connected: The power of the social media ecosystem. Business Horizons, 54(3), p. 265-273.

Itaú Bba. (2018). Análise econômico financeira dos clubes de futebol brasileiros. Recuperado em 24 novembro, 2018, de https://www. itau.com.br/_arquivosestaticos/itauBBA/Analise_Clubes_Brasileiros_Futebol_Itau_BBA.pdf.

Ibope Repucom. (2018). Ranking digital dos clubes brasileiros. Recuperado em 08 dezembro, 2018, de http://www.iboperepucom. com/br/rankings/ranking-digital-dos-clubes-brasileiros-dez2017/.

Ibope Repucom. (2017). Ranking digital dos clubes brasileiros. Recuperado em 04 janeiro, 2019, de http://www.iboperepucom. com/br/rankings/ranking-digital-dos-clubes-brasileiros-de-dezembro2016//.

Kaplan, A.M.; \& Haenlein, M. (2010). Users of the world, unite! the challenges and opportunities of social media. Business Horizons, 53(1), p. 59-68.

Lee, L.F; Hutton, A.P.; \& Shu, S. (2015). The role of social media in the capital market: evidence from consumer product recalls. Journal of Accounting Research, 53(2), p. 367-404.

Leite, M., Bambino, A. C., \& Hein, N. (2017). Relação Entre Política De Dividendos E Desempenho Econômico Financeiro Em Empresas Brasileiras E Chilenas. Revista de Gestão, Finanças e Contabilidade, 7(1), p. 205-221.

Leoncini, M. P.; \& Silva, M. T. da. (2005). Entendendo o futebol como um negócio: um estudo exploratório. Gestão \& Produção, 12(1), p.11-23

Lobato, P. L.; Dias, T. R.; \& Teixeira, M. M. (2012). Futebol: A importância das ferramentas de gestão para o sucesso do negócio. Motricidade, 8(2), p. 240-24

Mangold, W. G., \& Faulds, D. J. (2009) Social media: the new hybrid element of the promotion mix. Business Horizons, 52(4), 357-365.

Marteleto, R. M. Análise de redes sociais - aplicação nos estudos de transferência da informação. (2001). Ciência da Informação, 30(1), p. 71-81.

Meenaghan, T. (2001). Understanding sponsorship effects. Psychology \& Marketing, 18(2), 95-122.

Miragaia, D. A. M.; Ferreira, J. J. M.; \& Ratten, V. (2017). O envolvimento estratégico de stakeholders na eficiência das organizações desportivas sem fins lucrativos: De uma perspectiva de sobrevivência para a sustentabilidade. Brazilian Business Review, 14(1), p. 42-58.

Mokfianski, Karoline; \& Alvetti, Celina. (2017). O uso das redes sociais pelos clubes de futebol: o Facebook do Coritiba Foot Ball Club. Anais do Congresso Brasileiro de Ciências da Comunicação, Curitiba, PR, Brasil, 40.

Molina, G. (2014). Marketing deportivo digital: Pasión y negocios en la era tecnológica. Madrid: ESM LS.

Morgan, M. J.; \& Summers, J. (2008). Marketing esportivo. São Paulo: Thomson.

Mullin, B.J.; Hardy, S.; \& Sutton, W. (2004). Marketing esportivo. Porto Alegre: Artmed.

Paniagua, J., \& Sapena, J. (2014). Business Performance and Social Media: Love or Hate? Business Horizons, 57(6), p.719 -728.

Phillips, Robert. (2003). Stakeholder theory and organizational ethics. San Francisco: Berrett-Koehler Publishers Inc.

Phillips, R.; Freeman, R. E.; \& Wicks, A. C. (2003). What stakeholder theory is not. Business Ethics Quarterly, 13(4), p. 479-502.

Roberto J., \& Serrano, A. (2007). Desempenho empresarial, stakeholders e controlo estratégico, um estudo de caso. Conocimiento, innovación y empreendedores: camino al future, p. 2480-2495. Logroño: Universidad de Rioja.

Rosen, S. (1981). The economics of superstars. The American Economic Review, 71(5), p. 845-858.

Rubin, A.; \& Rubin, E. (2010). Informed investors and the internet. Journal of Business Finance \& Accounting, 37(7-8), p. 841-865.

Salles, L. R.; \& Castro Junior, J. L. P. (2015). O uso das redes sociais como estratégia de marketing nos clubes de futebol do Rio de Janeiro. Anais do Congresso Brasileiro de Ciências da Comunicação, Rio de Janeiro, RJ, Brasil, 38.

Safko, Lon; \& Brake, David K. (2009). The social media bible: tactics, tools and strategies for business success. John Wiley \& Sons Inc.

Scharf, E. R. (2010). O patrocínio do futebol como ferramenta do marketing esportivo para a construção de brand awareness. Revista Economia \& Gestão, 10(23), p.80-99.

Silva, C. V. D. G. F., \& Campos Filho, L. A. N. (2009). Gestão de clubes de futebol brasileiros: fontes alternativas de receita. Sistemas \& Gestão, 1(3), 195-209.

Silva, E. C. da, \& Silva, C. C. da. (2015). Naming Rights no Contexto Brasileiro: patrocínio de centros esportivos. Revista de Administração do UNIFATEA, 11(11), p.55-70.

Silveira, A.; Yoshinaga, C.; \& Borba, P. (2005). Crítica à teoria dos stakeholders como

função-objetivo corporativa. Caderno de Pesquisas em Administração, 12(1), p. 33-42.

Siqueira, J. P. L.; Pajanian, F.; \& Telles, R. (2015). Identificação e categorização dos stakeholders de um clube de futebol profissional brasileiro. Revista Ibero-Americana de Estratégia, 14(3), p. 15-31.

Sousa, J. C. de; Silva, P. M. M. da; Pinto, F. R.; Nascimento, A. L., \& Rabelo, L. N. (2018). A influência das redes sociais nas decisões de compras. Revista Gestão em Análise, 7(1), 118-127. 
Sports Value. (2018). Finanças dos clubes brasileiros em 2017. 2018. Recuperado em: 24 novembro, 2018, de http://www.sportsvalue.com.br/wp-content/uploads/2018/05/Sports-Value-Financas-Clubes-Brasileiros-Maio18.pdf.

Sšderman, S.; \& Dolles, H. (2013). Handbook of research on sport and business. Cheltenham: Edward Elgar.

Vincent, W. J.; \& Weir, J. P. (2012). Statistics in kinesiology. (4. ed) Champaign: Human Kinetics Publishers. 